\title{
EL TEATRO DURANTE LA GUERRA CIVIL ESPAÑOLA EN EL FRENTE Y LA RETAGUARDIA DE LA ZONA REPUBLICANA
}

\author{
MARIO MARTín GiJÓN \\ Universidad de Extremadura
}

\section{INTRODUCCIÓN}

Para situar la literatura dramática y su escenificación durante la guerra civil, hay que partir de una dicotomía que, a mi juicio, resulta operativa para todos los géneros, entre el espacio de producción del frente y el espacio de producción de retaguardia. ${ }^{1}$ Esta división se comenzó a forjar desde las primeras semanas de guerra, al haber escritores que se enrolaban como milicianos y otros que desempeñaban

\footnotetext{
${ }^{1}$ Recibido: 25/VII/2009 Aceptado: 10/IX/2009

En este trabajo se aplica un concepto espacial del "campo literario" como todo el conjunto de agentes que intervienen en la producción, difusión y valoración de la obra literaria: escritores, editores y público, que en el caso del género teatral incluye, naturalmente, actores, directores de escena, escenógrafos, etc. Dentro de este campo pueden acotarse distintos espacios de producción según las relaciones entre determinados autores, actores y público. Vid. P. Bourdieu, Les règles de l'art. Genèse et structure du champ littéraire, París, Éditions du Seuil, 1992.
} 
labores administrativas o de propaganda en la retaguardia. Como hiciera notar Serge Salaün: "pertinente parece la distinción entre teatro de la retaguardia y teatro militar: la diferencia entre los públicos acarrea un funcionamiento diferente del discurso teatral". ${ }^{2}$ A pesar de que el crítico francés hiciera esta distinción hace ya dos décadas, en los más recientes estudios sobre el teatro de la guerra prácticamente se sigue sin tener en cuenta esta separación, lo cual repercute negativamente en el análisis de los distintos fines y estrategias dramáticas recurrentes en ambos espacios. ${ }^{3}$

\section{El TEATRO DEL FRENTE, O LA ESCENIFICACIÓN DE LAS NECESIDADES DE LA GUERRA.}

Desde el primer momento, por la peculiar efervescencia cultural en las milicias republicanas, con las campañas de alfabetización y los propósitos didácticos y adoctrinadores, el teatro encontró un terreno muy favorable. ${ }^{4}$ Ya las abundantes arengas $\mathrm{y}$, sobre todo, los recitales de poemas frente a las tropas presentaban claros rasgos teatrales $\mathrm{y}$, de hecho, algunas obras podían ser definidas, según la situación de comunicación, como poemas o como pequeñas obras de teatro, lo cual muestra la hibridación de géneros que fue uno de los rasgos más inconfundibles de la literatura del frente. ${ }^{5}$ Por ejemplo, la pieza Monólogo del fusil de Herrera Petere, aparece en una versión reducida (22 versos) en la recopilación Guerra viva. Romances (1938), y en su versión más extensa (86 versos) es incluido en un librito de Teatro para combatientes con la acotación "Para recitar disfrazado de fusil".

\footnotetext{
2 S. Salaün, La poesía de la guerra de España, Madrid, Castalia, 1985, p. 118.

3 Por ejemplo en la, por lo demás, muy útil recopilación de N. Dennis y Emilio Peral Vega, (eds.), Teatro de la Guerra Civil: el bando republicano, Madrid, Espiral/Fundamentos, 2009.
}
4 Jim McCarthy señala como, por ejemplo, la sección de teatro de Altavoz del Frente estaba encuadrada en un proyecto mucho más amplio: "The work of Altavoz del Frente illustrates how [...] drama's role emerged as an element of diverse cultural, educational and propaganda programmes. The theatre sec- tion, for example, must be considered alongside the organization's other aspects, such as music, broad- casting, exhibitions, cinema and political education conferences". J. McCarthy, Political Theatre during the Spanish Civil War, Cardiff, University of Wales Press, 1999, p. 43.

5 Fue Gonzalo Santonja el primero en llamar la atención sobre esta ambivalencia de los romances: "puede deducirse que al menos algunos romances superaban el nivel de las simples recitaciones, encontrándose en los límites inciertos que separan a las composicones poéticas puras de las formas tetrales más elementales". G. Santonja, "Herrera Petere y el teatro de agitación", Triunfo (2-VII-1977), p. 53. Fernández Soria menciona que "[l]os montajes escénicos de poemas también fueron práctica de la Sección de Teatro del Altavoz del Frente". J. M. Fernández Soria, Educación y cultura en la guerra civil, Valencia, NAU Llibres, 1984, p. 85. Luis Miguel Gómez Díaz habla de "poemas -representables- de Rafael Alberti, retomados a principios de septiembre de 1936 en Madrid, poemas de Emilio Prados, publicados, de Pedro Garfias, de Antonio Porras, de Antonio Aparicio". L. M. Gómez Díaz. Teatro para una guerra [1936-1939]. Textos y documentos. Madrid, Centro de Documentación Teatral, 2006.

6 Vid. respectivamente J. Herrera Petere, Obras Completas. Poesía I, edición de Narciso Alba Hervalejo, Guadalajara, Diputación de Guadalajara / Junta de Comunidades de Castilla-La Mancha, 2007, p. 39; y J. Herrera Petere, Teatro para combatientes. El fusil. Torredonjil. La voz de España, prólogo de José Esteban, Madrid, Hispamerca, 1977, pp. 13-15. 
Por otra parte, el teatro producido en el espacio del frente, para el cual haría fortuna el sintagma "teatro de urgencia" acuñado por Rafael Alberti,7 se caracterizó, según definió Jim MacCarthy, por una directa relación temática con la problemática de la guerra, una intención adoctrinadora respecto a la audiencia y una función performativa respecto a ésta. ${ }^{8}$ Es decir, los principales rasgos diferenciales de este teatro (no sólo frente al teatro prebélico, sino, como veremos, al producido y representado en la retaguardia) tienen que ver con la relación entre acción representada y espectadores. ${ }^{9}$ Al mismo tiempo, conllevan una definición de literatura totalmente heterónoma y sometida a los fines prácticos de la guerra.

$\mathrm{Y}$ es que este teatro provenía directamente de los intentos de un teatro 'proletario', puestas en práctica durante los años treinta. Así, las primeras iniciativas de un teatro plenamente adscribible al espacio de producción en el frente surgen de Altavoz del Frente:

Formados los grupos teatrales que van a trabajar en los frentes, cuarteles, hospitales, centros obreros, etc., hacen falta obras. ALTAVOZ DEL FRENTE las pide a los autores, a los escritores antifascistas, a cuantos piensan colaborar con él, obreros e intelectuales. Las obras deben ser muy cortas, de quince o veinte minutos de duración necesariamente, de lucha contra el fascismo y exaltación del heroísmo popular. Enviadlas al local del Altavoz del Frente, Alcalá, $62 .{ }^{10}$

El grupo de teatro de Altavoz del Frente había sido impulsado por César e Irene Falcón, responsables del grupo "Nosotros", que se habían inspirado por las prácticas teatrales que conocieron al visitar la Olimpíada de Teatro Proletario en Moscú en 1932 y que incluso durante el "bienio negro" había continuado representando obras de tema revolucionrio. Por ello, según el periodista coetáneo Antonio Otero Seco, "surgió ya con pujanza y perfil de cosa lograda y madura, aupado por el entusiasmo de sus

\footnotetext{
7 R. Alberti, “Teatro de urgencia”, Boletín de Orientación Teatral, 1 (15-II-38), p. 1.

8 "First, the play's content will derive specifically from the Civil War. Second, the direct intention of the play will be to encourage in the spectator a 'correct' pro-Republican ideological position. Finally, through the achievement of the second criterion, the play will seek to inculcate values and delineate perspectives which will nurture the spectators as an ideal Republican combatant or citizen". J. McCarthy, op. cit, p. xviii.

9 "The definition emphasizes [...] the relationship between stage and spectator during performance. Dramatized material which has a specific basis in the conflict is ordered so as to exert, with a high degree of focused intention, a particular influence upon the audience which encourages certain habits, certain ways of thinking which correspond to the ideological aims of the Republican camp". McCarthy, op. cit, Ibid. Según Francisco Mundi Pedret, "la arenga se diluye en la emoción que produce [...] cuando Ontañón, por ejemplo, se refiere a los bombardeos de Madrid, se dirige a quienes son bombardeados. Por lo tanto "las obras del teatro de urgencia" no son obras analíticas, sino actos insertos en la dinámica del hecho histórico y, naturalmente, en un determinado campo ideológico". F. Mundi Pedret, El teatro de la guerra civil, Barcelona, PPU, 1987, p. 22.
}

10 “Altavoz del Frente. Teatro para los combatientes". El Sol (21-X-1936), p. 18. 
organizadores y por el fervor del pueblo combatiente". ${ }^{11}$ La sección teatral del Altavoz dependerá del Quinto Regimiento y estará constituida por dos grupos de “Guerrillas del Teatro" (no confundir con las Guerrillas posteriormente creadas) que representaba en cuarteles, hospitales y avanzadas del frente, y un tercer grupo, con mayores medios y dirigido por el actor y director Manuel González que llevó el nombre de “Teatro de Guerra" y que actuaba en Madrid. ${ }^{12}$ Dentro de este espacio de producción del frente, destacan también los "Cuadros Artísticos" adscritos a muchas Brigadas, el "Teatro Popular" dirigido por Luis Mussot (dramaturgo y comisario de guerra, cuya obra dramática durante la guerra es una de las más sólidas, y está aun por recuperar), los grupos "Curva”, “Luis de Sirval”, ligado al Socorro Rojo Internacional, “Arte y Cultura", dependiente de la Sección de Propaganda del PSOE, o la compañía de Benito Cebrián, así como, en Cataluña, los “Elencs de guerra".

Con la conformación del Ejército Popular, será el Comisariado de Guerra el organismo que centralice e impulse este teatro, proclamando la necesidad de crear y representar piezas de "breve teatro político antifascista", siempre con un claro objetivo relacionado con los problemas de los soldados. Puede hablarse de un "teatro aplicado", ${ }^{13}$ tanto a la enseñanza de cuestiones prácticas ${ }^{14}$ como, sobre todo, de consignas políticas pues era evidente, según Antonio Aparicio (poeta y dramaturgo, autor de la pieza Los miedosos valientes), "la utilidad de esta especie de teatro, hecho con obras cortas en las que se escenifica una consigna de guerra, un episodio aleccionador, se ha podido comprobar, de manera práctica, en todos los frentes". ${ }^{15}$ Por poner algunos ejemplos, en El refugiado, de Miguel Hernández o Torredonjil, de Herrera Petere, se ilustra la necesidad de la fraternización entre soldados y campesinos.

11 A. Otero Seco, “¡Aquí, Madrid, Altavoz del Frente!”, Mundo Gráfico (1-X-1936), p. 8. Agradezco a Mariano Otero San José haberme enviado fotocopia de éste y otros artículos escritos por su padre durante la guerra.

12 Ya por el hecho de representar en Madrid, en un teatro tradicional y no al aire libre, este tercer grupo se veía frente a unas condiciones diferentes. El 22 de octubre el "Teatro de Guerra" se presentó por primera vez con tres obras: Así empezó..., de Luisa Carnés, con decorados del dibujante Puyol, Bazar de la providencia, escrita por Alberti varios años antes, con decorados de Izarra, y La conquista de la prensa, escrita por Irene Falcón en 1934, con decorados de Viejo. Sólo la obra de Carnés, que recordaba las primeras horas del 19 de julio, tenía como motivo directo la guerra que se estaba viviendo en España. Posiblemente por ello, al terminar el espectáculo, César Falcón tuvo que explicar en una alocución al público el significado político de las obras representadas. Vid. R. Marrast, El teatre durant la guerra civil espanyola. Assaig d'història i documents, Barcelona, Edicions 62, 1978, p. 25.

13 Vid. Serge Salaün, "Le "vers appliqué" pendant la guerre d'Espagne", Mélanges de la Casa de Velásquez, VIII (1972), p. 525-560.

14 Así, la mencionada Monólogo del fusil de Herrera Petere sirve para enseñar el correcto cuidado y correcto manejo del arma a los reclutas novatos y en iHacia la victoria! de García Narezo, se muestra el comportamiento adecuado bajo el fuego enemigo.

15 A. Aparicio, "El teatro en nuestro ejército", Comisario, 4 (diciembre de 1938), p. 50. Vid. Comisario, 1938-1939, edición facsímil, edición e introducción de J. L. García Martín, Mérida, Editora Regional de Extremadura, 2003. 
A medida que avanza la guerra, sin embargo, el tema predominante es el de la lucha contra el derrotismo, que comenzaba a calar en la moral de los soldados. Invariablemente, en estas obras, como El bulo, de Santiago Ontañón, El café... sin azúcar, de Pablo de la Fuente, La voz de España, de Herrera Petere, o las anónimas jAplastar a Franco! y Lección y escarmiento del derrotismo, quien hace comentarios derrotistas es indefectiblemente un traidor, un quintacolumnista emboscado. ${ }^{16}$ Estas obras, por tanto, tienen como objetivo, en primer lugar, fomentar la abnegación de los soldados, en unos momentos especialmente difíciles y, en segundo, reforzar la censura contra los ataques al gobierno y a las decisiones militares.

Es característico de estas piezas de urgencia destinadas al frente el hecho de que no hay rastro de idealización de los combatientes, algo lógico pues éstos debían poder sentirse identificados. Era la misma tónica que perseguían, por ejemplo, obras como Hay que evitar ser tan bruto como el soldado Canuto, de la cual haría Luis Mussot una adaptación teatral. ${ }^{17}$

En cuanto a la función performativa respecto al público, en pocas obras es tan marcada como en una de las primeras creaciones del frente, la anónima 4 batallones de choque, creada y escenificada en noviembre de 1936, en los días más dramáticos de la defensa de Madrid..$^{18}$ La obra presenta la cola de un cine, en la que se exponen las diferentes actitudes frente a la guerra, incluyendo los argumentos de La Niña Cursi, El Burgués y El Artista, así como el de algunos emboscados "con carnet sindical". En la tercera y última escena, los obreros conscientes, El Obrero Alistado y La Obrera Alistada rompen la convención escénica para dirigirse directamente al público, animándole a alistarse. El colofón de la obra es la partida del camión, supuestamente hacia el frente, con lo que se liquida la acción teatral y se pasa, sin solución de continuidad, a la acción real. Esta integración con el público es perseguida en muchas obras, por ejemplo en la obra de Max Aub, ¿Qué has hecho hoy para ganar la guerra?, donde los actores preguntan a otros actores situados estratégicamente entre el público, diluyendo así las fronteras entre realidad y escena. ${ }^{19}$

16 Como describe Gregorio Torres Nebrera, en estas obras, invariablemente "se produce un enfrentamiento entre dos grupos, el de los quintacolumnistas y el de los fortalecidos por una moral de sacrificio, de lealtad y de victoria en la guerra que padecen y soportan con ejemplar entrega. Los primeros acaban inevitablemente descubiertos y rechazados por los segundos (la necesaria voz de alerta que se quería transmitir desde el tablado al espectador) y se procura siempre que del caso particular propuesto en la representación se deduzca el mensaje general que el público debe aprender a toda costa". G. Torres Nebrera. "Las guerrillas del teatro (urgencia, propaganda, compromiso)". ADE Teatro, 77 (octubre 1999), pp. 144-153.

17 El texto se incluye en el CD del libro de Díaz Gómez, op. cit.

18 Recogida en N. Dennis y E. Peral Vega, op. cit., pp. 109-115.

19 Vid. M. Aub, ¿Qué has hecho hoy para ganar la guerra?, en Primer Teatro. Obras Completas, vol. VII-A, edición, estudio introductorio y notas de Josep Lluís Sirera, Valencia, Biblioteca Valenciana, 2002, pp. 


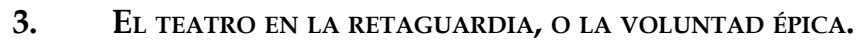

En cuanto al espacio de retaguardia, como demostró sobradamente Robert Marrast, durante la guerra civil se vio una mayoritaria permanencia de formas teatrales convencionales. Entre las iniciativas más innovadoras, en el espacio de retaguardia, la Alianza de Intelectuales Antifascistas irá imponiendo una cierta predominancia, sobre todo a partir de que surja el grupo "Nueva Escena" como sección de teatro de la Alianza, impulsado por María Teresa León y Rafael Alberti, y que a principios de octubre de 1936 logra que se le ceda para sus representaciones uno de los mejores escenarios de la capital madrileña, el Teatro Español, del que Rafael Dieste fue nombrado director.

Desde su estreno el 20 de octubre, “Nueva Escena” persiguió un teatro de calidad que aunara los esfuerzos de escritores y pintores, la mayoría de ellos pertenecientes a la Alianza. Así, en esta primera sesión se representaron: La llave, de Sender, con decorados de Arturo Souto; Al amanecer, de Rafael Dieste, con decorados de Santiago Ontañón, y Los salvadores de España, de Alberti, con decorados de Miguel Prieto. ${ }^{20}$

Otros autores buscaron de manera aislada la creación de un "teatro popular" como José Antonio Balbontín, ${ }^{21}$ quien fue uno de los pioneros en la creación de un teatro de guerra, con su estreno de El Cuartel de la Montaña, el 15 de septiembre de 1936 en el Teatro Maravillas, obteniendo un gran éxito prolongado en El Frente de Extremadura y Pionera, estrenadas en el mismo teatro en octubre de 1936, "hasta que el teatro fue materialmente cerrado por los bombardeos" de la aviación fascista, según el propio Balbontín. ${ }^{22}$

El avance de las tropas fascistas supuso la brusca interrupción de la formación de un espacio de producción literaria en Madrid, desplazándose éste, al compás de las transformaciones en el campo de poder, a Valencia. Frente a la función performativa del teatro en el frente, que apelaba directamente al público, con el que a veces la obra se fundía, y que tenía un final abierto que había de ser completado en la vida real por los soldados convertidos en espectadores, obras como las de Balbontín sirven para canonizar momentos fundacionales de la "épica" del pueblo y por ello resultaba en comparación estático y relativamente autónomo.

\section{5-311.}

20 Marrast, op. cit., pp. 23-24.

${ }_{21}$ Sobre el teatro de guerra de Balbontín, vid. A. L. Larrabide, "El teatro de José Antonio Balbontín", Dioniso. Revista para la investigación, creación y crítica teatral, 3 (2007), pp. 11-22.

22 J. A. Balbontín. La España de mi experiencia. Reminiscencias y esperanzas de un español en el exilio, introducción de J. Rubiales Torrejón, prólogo de A. L. Larrabide, Sevilla, Centro de Estudios Andaluces, 2008, p. 209. 
Las actividades teatrales de la Alianza se verían interrumpidas primero por las batallas en torno a Madrid y después ralentizadas por el viaje de María Teresa León y Rafael Alberti a la Unión Soviética y la tensa situación política provocada por la crisis del gobierno de Largo Caballero. Con la consolidación del gobierno de Negrín, las iniciativas oficiales a favor del teatro cobraron un nuevo impulso y el 22 de agosto de 1937 se creó el Consejo Central del Teatro, destinado a ser un "órgano adecuado que oriente y encauce las actividades teatrales" ${ }^{23}$ y cuya composición resulta muy significativa de la relación de fuerzas dentro del espacio de producción de la retaguardia, íntimamente ligado al campo de poder: El presidente era el director general de Bellas Artes, Josep Renau; los vicepresidentes, Antonio Machado y María Teresa León; secretario, Max Aub; y entre sus miembros podemos encontrar a autores consagrados como Jacinto Benavente, a los dos críticos de teatro más prestigiosos desde años atrás (Enrique Díez Canedo y Cipriano Rivas Chérif, quienes además desempeñaban funciones diplomáticas), autores y realizadores innovadores como Alejandro Casona, Miguel Prieto, Manuel González y a músicos como Enrique Casal Chapí o Martínez Allende.

De María Teresa León y Rafael Alberti partieron las iniciativas de mayor envergadura, con la creación del "Teatro de Arte y Propaganda" (cuyo nombre es todo un manifiesto del discurso que se había impuesto durante la guerra, en el que el arte y la propaganda resultaban perfectamente compatibles) que presentó su primer espectáculo el 10 de septiembre de 1937, en un escenario relativamente lujoso como era el Teatro de la Zarzuela. La selección de obras en esta sesión inaugural es significativa de una voluntad de autonomía, no directamente relacionada con las cuestiones prácticas de la guerra. Se representaron Los títeres de cachiporra, obra aún inédita de Lorca, con figurines de Santiago Ontañón y música de Jesús G. Leoz y La cacatúa verde de Arthur Schnitzler. Asimismo, en las obras del "Teatro de Arte y Propaganda" se ve una clara voluntad de enlazar con la tradición, por ejemplo, con la adaptación de la Numancia de Cervantes, a cargo de Rafael Alberti. El tema de esta obra resultó polémico pues, como criticaron algunos, en la obra de Cervantes, al fin y al cabo, los numantinos resultaban vencidos. Sin embargo, lo que buscaba Alberti era la presentación de una imagen abnegada, heroica, que se irá imponiendo en el teatro de retaguardia. La imagen idealizada de los milicianos que aparece en obras como Sombras de héroes de Germán Bleiberg o El Cuartel de la Montaña de Balbontín, sería imposible de presentar, con pretensiones de realismo, frente a un auditorio de milicianos. ${ }^{24}$

\footnotetext{
23 Véase el texto completo del decreto en Marrast, op. cit., pp. 245-246.

24 Como señala Jim McCarthy: "It is surely significant, however, that in teatro de urgencia which is known to have been presented before troop audiences, and whose principal focus is the miliciano, idealized images of combatants are eschewed in favour of a more broadly realistic exploration of military life and its obligations". Op. cit., p. 138.
} 
Junto a la idealización de los milicianos en las producciones más ambiciosas, en el espacio de retaguardia aparecen también numerosas obras satíricas, que presentan una imagen paródica de los sublevados. En obras como Radio Sevilla, de Alberti, o Nuevo retablo de las maravillas, de Rafael Dieste, ${ }^{25}$ en las que respectivamente Queipo de Llano y "el General", son vapuleados por los milicianos en un final propio de guiñol, género al que estas obras de teatro fueron fácil y frecuentemente adaptadas. En otra obra similar, ¡Arriba Italia! de Herrera Petere, los personajes falangistas aparecen como afeminados, cobardes y dispuestos a vender su patria al extranjero, siendo interrumpidas sus maquinaciones por la irrupción de unos milicianos que los someten fácilmente. Una acción similar presenta la anónima Gudalajara-Italia. ${ }^{26}$ Estas representaciones, como es lógico, tenían una mejor acogida en la retaguardia, por la función de degradación del enemigo, que en el frente, donde se era consciente de su potencial.

El Consejo Central del Teatro editaba un Boletín de Orientación Teatral, del que aparecieron seis números entre el 15 de febrero y el 1 de junio de 1938, con una función, como su título indica, claramente prescriptiva. Ya consolidada la autoridad de los autores "reconocidos" para imponer las directrices sobre la creación en los espacios de producción tanto del frente como de la retaguardia, el ensayo de Alberti presentaba un carácter directivo que habría sido inimaginable a principios de la guerra.

A finales de 1937 se produce una importante iniciativa de penetración del espacio de producción cultural de retaguardia en el espacio del frente: la creación de las "Guerrillas del Teatro", que retomaban una iniciativa que ya había existido, de forma menos organizada, en el Quinto Regimiento, y que fueron dirigidas por María Teresa León y aprobadas por decreto del 14 de diciembre de 1937. ${ }^{27}$ Resultó una creación afortunada ya que en los meses siguientes las transformaciones dentro del campo de poder y concretamente el traspaso del Ministerio de Instrucción Pública de Jesús Hernández (PCE) a Segundo Blanco (CNT) en enero de 1938, con las subsiguientes dimisiones de los Subsecretarios Wenceslao Roces y Josep Renau iban a tener importantes repercusiones en los apoyos externos a la producción cultural, con una disminución del apoyo a las actividades de la Alianza, uno de cuyos primeros resultados sería la retirada del permiso concedido al "Teatro de Arte y Propaganda" para actuar en el Teatro de la Zarzuela, que se cedió a otras compañías. ${ }^{28}$

25 Vid. R. Dieste, “Nuevo retablo de las maravillas”, Hora de España, 1 (enero 1937), pp. 65-79.

26 Ambas obras, hasta ahora inéditas, serán incluidas en la edición del Teatro de José Herrera Petere que está preparando Manuel Aznar Soler, dentro de las Obras Completas que han comenzado a editar conjuntamente la Diputación Provincial de Guadalajara y la Junta de Comunidades de Castilla-La Mancha.

27 Véase el texto del decreto en Marrast, op. cit., pp. 259-260.

28 Vid. F. Doménech, “El teatro en la Guerra Civil española”, ADE Teatro, 77 (octubre 1999), p. 82. 
En estas condiciones, las Guerrillas del Teatro se mantendrán en el espacio de producción del frente, menos condicionado por los movimientos en el campo de poder, y dependientes, por su parte, de un verdadero poder paralelo en la España republicana, el de las unidades militares, en la mayoría de las cuales afianzaba su control el PCE durante esta fase de la guerra. Las Guerrillas del Teatro del Ejército del Este actuarían, por ejemplo, en posiciones del frente de Gandesa entre marzo y abril de 1938, una zona en la que se encontraban las unidades militares más combativas y mejor organizadas, que además eran las mejor controladas por el PCE. Varias de las piezas representadas serían recopiladas en el libro Un teatro de guerra (Las Guerrillas del Teatro), publicado en Barcelona en 1938 por la editorial Nuestro Pueblo. Entre las piezas recogidas, hay varias que tematizan la relación entre los soldados y los campesinos de los pueblos cercanos al frente, por ejemplo Pueblos de vanguardia y Defendemos la tierra. ${ }^{29}$ Como ha señalado Jim McCarthy, cabe hacer una distinción entre las Guerrillas del Teatro del Ejército del Este, que actuaron en una zona entonces plenamente bajo ataque fascista, y las otras Guerrillas del Teatro que actuaron en el Centro y en otras zonas más tranquilas. Mientras que las obras de las primeras apenas se diferencian de las creadas por los escritores militarizados y fomentadas por el Comisariado de Guerra, las representadas por las Guerrillas del Teatro en los pueblos del Guadarrama (en 1938 una zona relativamente pacífica) presentaban una menor insistencia en la "consigna" que se trataba de transmitir, es decir, con obras creadas con cierta voluntad artística y que podían ser mejor aprecidas "en sí", frente a la concepción de la obra como desencadenante performativo de acciones por parte del público, que debería concretarse después de la representación, en la vida real. ${ }^{30}$ Las “Guerrillas" del Ejército del Centro representaron a veces obras desconectadas de la problemática bélica inmediata, que recuerdan, tanto en la selección como en la percepción del "pueblo" a las actuaciones del "Teatro del Pueblo" de las Misiones Pedagógicas o La Barraca. Resulta curioso que María Teresa León, que en 1933 criticó acerbamente a estas dos compañías, diciendo que "no supieron emplearse políticamente, con la política que el espectador quería encontrar", ${ }^{31}$ elogiara años después la actividad de las Guerrillas

\footnotetext{
29 Para un análisis de estas piezas, vid. G. Torres Nebrera, op. cit.

30 "The feeling of actualité is intense in those works which have survived from the repertoire of the Army of the East's Guerrillas del Teatro [...]. By contrast, the other Guerrillas del Teatro established by the government ministry, that of the Army of the Centre, presented a somewhat different teatro de urgencia, focusing less on aggressive, slogan-like work than on satire and rather more conventional examples of the one-act play". J. McCarthy, op. cit., p. 51. El crítico galés atribuye este distinto carácter, además de un factor extraliterario como es la mayor actividad del Frente del Ebro, a un factor interno: la subordinación a la Alianza de Intelectuales, institución por esa época plenamente de retaguardia: "That this should be so is undoubtedly due to the leading role of the Alliance in the creation of the Guerrillas del Teatro". Ibid.
}

31 M. T. León, "El teatro internacional. Panorama de los teatros de Moscú", Heraldo de Madrid (23 de mayo de 1933), p. 6. Apud. M. T. León, Obra dramática y escritos sobre teatro, edición de Gregorio Torres Nebrera, Madrid, ADE, 2003, p. 378. 
del Ejército del Centro en unos términos que recuerdan al apoliticismo que achacara a aquellas: "por todos los lugares libres se instaló un público con las manos sucias y las caras sudorosas y los trajes de faena, engrasados [...] para oír los graciosos versos de Lope de Vega y las afortunadas ocurrencias de Chejov, de Alberti, de Santiago Ontañón". 32

Otro intento de extender el discurso de retaguardia a los frentes sería realizado por los escritores de Hora de España que, frente a los teatros de las unidades militares, intentaron ayudar a relanzar La Barraca, nombrando como director de ella a Manuel Altolaguirre, que dirigiría a algunos estudiantes de bachillerato que aún no estaban en edad militar. La iniciativa, totalmente desfasada ya respecto a la problemática de la guerra, fracasaría, pero es significativa del discurso del espacio de producción de retaguardia, 'conservador' en sus formas comparado con el teatro del frente. Como hemos podido comprobar, el teatro en el espacio de producción del frente tiene unas características nuevas, basadas en la relación con el público y su función performativa, muy distintas a las iniciativas anteriores a la guerra. Así, La Barraca fracasaría lógicamente en su adaptación a las circunstancias de la guerra. Si bien actuaría en algunas ocasiones, por ejemplo, en el verano del 36 frente a la Brigada Motorizada de Hierro, solicitada por su jefe, Gustavo Durán, que había sido amigo de Lorca, sólo actuó algunas veces más.

\section{CONCLUSIÓN}

En definitiva, el teatro durante la guerra civil española ejerció en el frente y la retaguardia dos funciones muy distintas, sirviendo en el frente como medio de educación y adoctrinamiento político de los soldados, mientras que en la retaguardia servía para reforzar la moral de los espectadores y su confianza en la victoria. Estas funciones se desenvolvían en escenarios muy distintos, teniendo el teatro de retaguardia a su disposición los edificios que habían albergado el teatro anterior a la guerra, mientras que en el frente prácticamente todo se confiaba a la habilidad e improvisación de los actores. Paradójicamente, la riqueza de medios hizo que el teatro de retaguardia no lograra grandes innovaciones, habiendo de competir, además, con el habitual teatro comercial, que gozaba el favor de muchos espectadores que preferían acudir al teatro para evadirse de la guerra durante unas breves horas. En cambio, a

${ }_{32}$ M. T. León, Juego limpio. Barcelona, Seix Barral, 1987, p. 167. Esta descripción y orientación 'clasicista' sorprende aún más viniendo de la misma autora que durante la guerra atacara duramente de nuevo la concepción apolítica del teatro de las Misiones Pedagógicas y de La Barraca, ya que carecían del "sentido político de lo que estas aldeas debían y querían ver". M. T. León, “La guerra, el teatro, la revolución y la industria", Boletín de Orientación Teatral, 3 (1938), p. 5. La selección de estas obras es una muestra de lo difícil que para un escritor resulta renunciar a apoyarse en una tradición que legitime incluso las iniciativas aparentemente más rupturistas. 
pesar de la tosquedad de alguna de sus piezas, el teatro del frente logró, con pocos medios, los fines a los que se destinaba, logrando una poco común fusión entre actores y espectadores. Sin duda, habría que valorar los "efectos de presencia", por encima de los de significado, ${ }^{33}$ en estas obras que, dentro de su contexto, cumplieron la función para la que habían sido creadas.

\section{Bibliografía}

Alberti, R., "Teatro de urgencia”, Boletín de Orientación Teatral, 1 (15-II-1938), pp. 1-2. ApARICIO, A., "El teatro en nuestro ejército", Comisario, 4 (diciembre de 1938), pp. 47-50, edición facsímil, edición e introducción de J. L. García Martín, Mérida, Editora Regional de Extremadura, 2003.

“Altavoz del Frente. Teatro para los combatientes". El Sol (21-X-1936), p. 18.

Aub, Max, Primer Teatro. Obras Completas, vol. VII-A, edición, estudio introductorio y notas de Josep Lluís Sirera, Valencia, Biblioteca Valenciana, 2002.

BALbontín, J. A. La España de mi experiencia. Reminiscencias y esperanzas de un español en el exilio, introducción de J. Rubiales Torrejón, prólogo de A. L. Larrabide, Sevilla, Centro de Estudios Andaluces, 2008.

Bourdieu, P., Les règles de l'art. Genèse et structure du champ littéraire, París, Éditions du Seuil, 1992.

Dennis, N. y E. Peral Vega, (eds.), Teatro de la Guerra Civil: el bando republicano, Madrid, Espiral/Fundamentos, 2009.

DoméneCH, F. “El teatro en la Guerra Civil española”, ADE Teatro, 77 (octubre 1999), pp. 75-85.

Dieste, R. “Nuevo retablo de las maravillas", Hora de España, 1 (enero 1937), pp. 65-79.

FERNÁNDEZ SORIA, J. M., Educación y cultura en la guerra civil, Valencia, NAU Llibres, 1984.

Gómez DíAz, L. M., Teatro para una guerra [1936-1939]. Textos y documentos, Madrid, Centro de Documentación Teatral, 2006.

Gumbrecht, H. U., Production of Presence. What Meaning Cannot Convey. Stanford, Stanford University Press, 2004.

Herrera Petere, J., Obras Completas. Poesía I, edición de Narciso Alba Hervalejo, Guadalajara, Diputación de Guadalajara / Junta de Comunidades de CastillaLa Mancha, 2007.

, Teatro para combatientes. El fusil. Torredonjil. La voz de España, prólogo de José Esteban, Madrid, Hispamerca, 1977.

33 Vid. H. U. Gumbrecht, Production of Presence. What Meaning Cannot Convey. Stanford, Stanford University Press, 2004. 
Mario Martín Gijón

Larrabide, A. L. “El teatro de José Antonio Balbontín”, Dioniso. Revista para la investigación, creación y crítica teatral, 3 (2007), pp. 11-22.

LEón, M. T., Obra dramática y escritos sobre teatro, edición de Gregorio Torres Nebrera, Madrid, ADE, 2003.

------, Juego limpio. Barcelona, Seix Barral, 1987

Marrast, R., El teatre durant la guerra civil espanyola. Assaig d'història $i$ documents, Barcelona, Edicions 62, 1978.

McCarthy, J., Political Theatre during the Spanish Civil War, Cardiff, University of Wales Press, 1999.

Mundi Pedret, F., El teatro de la guerra civil, Barcelona, PPU, 1987.

Otero Seco, A., “'Aquí, Madrid, Altavoz del Frente!”, Mundo Gráfico, 1-X-36, p. 8.

SAlAün, S., La poesía de la guerra de España, Madrid, Castalia, 1985.

--------, "Le "vers appliqué" pendant la guerre d'Espagne", Mélanges de la Casa de Velásquez, VIII (1972), p. 525-560.

SAntonjA, G., “Herrera Petere y el teatro de agitación”, Triunfo (2-VII-1977), pp. 52-53.

Torres Nebrera, G., "Las guerrillas del teatro (urgencia, propaganda, compromiso)”, ADE Teatro, 77 (octubre 1999), pp. 144-153. 